\title{
Tissue Segmentation in MRI as an Informative Indicator of Disease Activity in the Brain
}

\author{
Simon Vinitski, Ph.D., Carlos Gonzalez, M.D., Claudio Burnett, M.D., Feroze \\ Mohamed, M.S, Tad Iwanaga, M.S., Hector Ortega, M.D., Scott Faro, M.D.* \\ Department of Radiology, Thomas Jefferson University Hospital \\ 132 S. 10th Street, Room 1098, Main Building, Philadelphia, PA 19107 \\ 215-955-7293 (FAX) 215-955-5329 \\ * Department of Radiology, Medical College of Pennsylvania, Philadelphia, PA
}

\begin{abstract}
The presented tissue segmentation technique is based on a multispectral analysis approach. The input data were derived from high resolution MR images. Usually, only two inputs, proton density (PD) and T2weighted images, are utilized to calculate the 2D feature map. In our method, we introduced a third input, T1-weighted MR image, for segmentation based on 3D feature map. k-Nearest Neighborhood segmentation algorithm was utilized. Tissue segmentation was performed in phantoms, normal humans and those with brain tumors and MS. Our technique utilizing all three inputs provided the best segmentation $(p<0.001)$. The inclusion of $T 1$ based images into segmentation produced dramatic improvement in tissue identification. Using our method, we identified the two distinctly different classes of tissue within the same MS plaque. We presume that these tissues represent the different stages involved in the evolution of the MS lesions. Further, our methodology for measuring MS lesion burden was also used to obtain its regional distribution as well as to follow its changes over time. The segmentation results were in full accord with neuropsychological findings.
\end{abstract}

Key Words: MRI, Tissue segmentation, Multiple Sclerosis, brain tumor, 3D Feature Map

\section{Introduction}

The presented work deals with the application of tissue segmentation to characterize it both qualitatively and quantitatively using MR imaging. Previously, we utilized the method of Cline et al. who used probability and connectivity on two sets of images (proton density and T2-weighted) to segment brain tissues (1). We extended the segmentation algorithm into the third dimension. Many tissues (defined probabilistically) have overlapping MR signal intensities. We hypothesized that adding a third input image would make the separation of the clusters in a 3D feature space greater, with a resulting improvement in tissue segmentation. Initial results showed that segmentation image based on 3D feature map was better than or equal to the best segmented image based on 2D feature map (2). However, there was not enough data to perform quantitative analysis.

Since that time we have improved our methodology (e.g., move from probability model to k-Nearest Neighborhood). Initially, we tested this improved methodology in animals (experimental edema in cat brain). Results showed an inter-observer variability in the range of $7.6 \%-9.3 \%$ and intra-observer variability range was $8.3 \%-11.4 \%$, indicating high stability of the technique (3).

To determine volumetric accuracy of our technique a phantom study was also performed (4). Five samples which represented CSF, white/gray tissues, blood and fat, 
were utilized as a phantom. To establish volumetric accuracy, the volume of each sample was accurately measured prior to MR imaging. Results of volumetric phantom measurements show accuracy between 3 - 5\%.

In this study we had the following aims: a) to demonstrate the advantage of tissue segmentation based on 3D feature map over that based on 2D by quantitative means and in patients, particularly those with MS and, b) to investigate the effects of inclusion of T1-weighted MRI into the segmentation process of the brain tissues. Further, currently no reliable marker of the number of brain pathologies, such as MS activity, over time exists. Thus, the final aim of our study was to accurately measure regional lesion load as well as to follow its changes over time.

\section{Methods and Materials}

As we stated earlier, we have utilized a multispectral analysis approach (1). Three sets of MRI data were utilized in phantom and human experiments. Proton density and T2weighted fast spin echo and, as a third input, T1-weighted spin echo were used. Image matrix was up to $256^{2}$ and $3-5 \mathrm{~mm}$ slice thickness was used. A $1.5 \mathrm{~T}$ Signa GE scanner was utilized.

Humans were five normal, six with brain tumor, and thirteen patients with MS. From the brain MS patient population, three patients also undergo a second follow-up study one-half year later.

For segmentation, images were transferred to Sun Sparc Station 2. Usually, only two inputs, proton density (PD) and T2-weighted images, are utilized to calculate the 2D feature map. In our method, we introduced a third input, T1-weighted MR image, for segmentation based on 3D feature map. The qualified observer "seeded" tissue samples (40-50 samples/tissue) from three input images simultaneously. Later either three sets of image intensities (for 3D feature map) or any combination of two of them (for 2D feature map) were used for segmentation. Frequently, the most and least intense regions of the MS plaque were treated as two different tissues. Statistical filter narrowed sample distribution, thus, greatly reducing operator error in misclassified seeding. Anisotropic diffusion filter (5) was used for some correction of partial volume effects. Instead of probability model, as suggested by Clarke et al. (6), k-Nearest Neighborhood segmentation was utilized for both $2 \mathrm{D}$ and $3 \mathrm{D}$ feature map calculation. This data was used to create a stack of color-coded segmented images (one based on three inputs and three based on different combinations of two inputs) and up to eight tissues were classified. A connectivity algorithm (7) along with a dividing cube algorithm (8) constructs a surface of selected tissue(s). Finally, five board certified neuroradiologists ranked segmented images on a scale from 1 to 10 . Statistical analysis was performed using the Wilcoxon Matched-Pairs Signed-Ranks Test (9).

\section{Results}

In MS, Table 1 demonstrates the results of ranking for one segmentation based on 3D feature map (PD, T1, and T2) and three based on 2D. Results show segmentation based on $3 \mathrm{D}$ feature map was found to be far superior than any combination of $2 \mathrm{D}$ feature map (average rank $7.3, p<0.001$ ). We were surprised to find that the segmented image resulting from a combination of $\mathrm{PD} / \mathrm{T} 1$ was superior to two other methods based on $2 \mathrm{D}$ feature map: combinations of $\mathrm{PD} / \mathrm{T} 2$ and $\mathrm{T} 1 / \mathrm{T} 2$ (average rank 5.1 vs 3.7 and 3.4 $(p<0.01)$ respectively). Partial volume effects were visibly reduced. In addition, 
Table 1: Evaluation of segmentation based on $3 D$ and three $2 \mathrm{D}$ feature maps in MS

\begin{tabular}{|c|c|c|c|c|}
\hline \multirow{2}{*}{ Observers } & 3D & \multicolumn{3}{|c|}{ 2D } \\
\cline { 2 - 5 } & PD, T1,and T2 & PD and T2 & T1 and T2 & T1 and PD \\
\hline$\# 1$ & 6.69 & 3.38 & 3.23 & 4.23 \\
\hline$\# 2$ & 7.38 & 2.38 & 3.08 & 5.23 \\
\hline$\# 3$ & 7.38 & 4.31 & 4.15 & 5.00 \\
\hline$\# 4$ & 7.62 & 3.23 & 3.69 & 5.77 \\
\hline$\# 5$ & 7.53 & 3.38 & 4.31 & 5.38 \\
\hline Average & $\mathbf{7 . 3 2}$ & $\mathbf{3 . 3 4}$ & $\mathbf{3 . 6 9}$ & $\mathbf{5 . 1 2}$ \\
\hline
\end{tabular}

$\mathrm{PD}, \mathrm{T} 1$ and $\mathrm{T} 2=$ proton density, $\mathrm{T} 1$ - and $\mathrm{T} 2$-weighted images, respectively. Ranking ranges from 1 to 10 , where 10 is the best in lesion identification.

accuracy of phantom volumetric measurements was improved by $54 \%$ as compared to the previous results (2).

Moreover, the inclusion of T1 based images into segmentation of MS patients produced dramatic improvement in tissue identification, particularly between different areas within the same plaque. There was no mix between color pixels belonging to both "tissues." The color (class) distributions closely resembled cluster pattern. While no statistical analysis of these two "tissues" was performed, this data, nonetheless, strongly indicates the possible existence of two different, at least from the MRI point of view, tissues within the plaque. We presume that these two different classes of tissue most likely represent the different stages involved in the evolution of the MS lesions, such as: edema, demyelination, as well as residual cystic formation.

Table 2. Region distribution of MS lesion (cc) in a patient

\begin{tabular}{|l|r|r|r|r|r|}
\hline & Frontal & Parietal & Temporal & Occipital & Total \\
\hline Skin and Fat & 11.76 & 5.19 & 8.59 & 10.25 & 35.79 \\
\hline Gray Matter & 196.27 & 159.36 & 127.47 & 88.70 & 571.80 \\
\hline White Matter & 104.78 & 101.14 & 52.75 & 59.39 & 318.06 \\
\hline CSF & 43.51 & 52.67 & 31.71 & 8.33 & 136.22 \\
\hline MS Lesion & 27.78 & 30.16 & 16.61 & 9.67 & 84.22 \\
\hline Vascularity & 31.72 & 15.61 & 25.00 & 12.34 & 84.67 \\
\hline
\end{tabular}

Reproducible and accurate measurements of regional lesion load (temporal, parietal frontal, occipital) were obtained in each patient (Table 2). The majority of lesions were identified in the parietal occipital lobes. When the MS load increased it was associated with brain atrophy represented by an increase in CSF volume as well as a decrease in brain normal tissues volume. This is illustrated in Table 3. Importantly, these results of segmentation were consistent with the patients' clinical and neuropsychological evaluations of the disease over a six-month period. 
Table 3. Changes in MS load (cc) with time

\begin{tabular}{|l|c|c|c|}
\hline \multicolumn{1}{|c|}{ Tissue } & Start & $\mathbf{6}$ months & Change \\
\hline Normal Brain & 786.1 & 740.8 & $-5.8 \%$ \\
\hline CSF & 145.0 & 171.8 & $+18.5 \%$ \\
\hline MS & 79.9 & 94.1 & $+17.7 \%$ \\
\hline Total Volume & 1011.0 & 1007.0 & $0.0 \%$ \\
\hline
\end{tabular}

\section{Discussion}

We do not have enough data to quantitatively evaluate the use of tissue segmentation in brain tumors. However, we gained some experience with investigation of brain MS activity using tissue segmentation based on 3D feature map.

Cerebral lesions in MS have been described as clinically relevant only in advanced stages of the disease. Unfortunately, their effects are not readily apparent on the standard neurologic examination, disability rating scales, or gross screening instrument. In contrast, MRI has shown lesions in the brain much earlier in the disease. Quantitative measurements were obtained originally by Rao et al. $(10,11)$ using a $2 D$ "region of interest" method resulting in obtaining the area encompassed by each tracing. These measurements were then correlated with the patient's cognitive dysfunction. Their results, as well as ours $(12,13)$, demonstrated that there is a direct correlation between brain lesion load and neuropsychological deficit. In another study using specific rating of lesions involvement by frontal, temporal and parieto-occipital regions we quantified the topographic distributions of lesions and consequent effects upon cognitive function (14). In this context, quantitative MRI measurement of brain lesions provides an excellent opportunity to study altered brain behavior. All of these studies were performed using manual tracing of the lesions. Unfortunately, such studies are not very accurate. In a recent study of MS, a multi-institutional group (15) found that in manual measurements inter- and intra-observer variability can be as much as $100-200 \%$; and effectively gave this task to one "selected" technologist.

Another important application of quantitative MRI measures is in the performance of therapeutic clinical trials. Due to the recurrent nature of MS lesions, and the fact that new lesions detected by MRI are neurologically silent, the results of therapeutical clinical trials are difficult to evaluate. Using MRI manual tracing of the lesions as an indicator of treatment effects, Paty et al. (15), studied successfully the effect of Interferon $1 \mathrm{~B}$ in MS patients. Other parallel studies using MRI as an indicator for disease activity have also been performed. However, based on our results, we believe that tissue segmentation analysis and resulting volumetric measurements of the lesions provides a more sensitive, accurate and, most importantly, stable correlation with the results of the neuropsychological testing and can be relatively easily repeated and compared.

In conclusion, the presented work shows that segmentation based on 3D feature map is vastly superior to that based on $2 \mathrm{D}$. Next, the results show that it is possible to apply a reasonably mature segmentation technique to the actual disease processes on the quantitative and useful level. The inclusion of T1-weighted information into our segmentation methodology allowed better characterization of MS plaque. The results also suggest that our method of tissue segmentation is a reliable technique to measure, at least, MS lesion burden. We have also shown that it can also be used as a surrogate marker for disease activity over time. Finally, this technique can also be a useful adjunct in the future performance of therapeutic clinical trials in MS and other diseases. Thus, further clinical evaluation of this technique in other brain abnormalities is warranted. 


\section{References}

1. Cline HE, Lorensen WE, Kikinis R, Jolesz F. Three-dimensional segmentation of MR images of the head using probability and connectivity. J Comp Assist Tomogr 1990; 14:1037-1042.

2. Vinitski S, Seshagiri S, Mohamed FB, et al. Tissue characterization by MR: data segmentation using 3D feature map. In: Vernazza $G$, Venetsanopoulos AN, Braccini C (eds), Image processing theory and applications. Amsterdam:Elsevier Science Publishers B.V., 1993; 325-328.

3. Vinitski S, Gonzalez C, Burnett C, Seshagiri S, Mohamed FB, Lublin FD, Knobler RL, Frazer G. Tissue segmentation by high resolution MRI: improved accuracy and stability. Proc. IEEE Eng. Med. Biol. 1994; 16:577-578.

4. Vinitski S, Gonzalez C, et al. Improved Intracranial Lesion Characterization by Tissue Segmentation Based on 3D Feature Map. Radiology 1994; 193(P):253.

5. Perona $P$, Malik J. Scalespace and edge detection using anisotropic diffusion. Proc IEEE Workshop on Computer Vision, Miami, FL 1987; 6-22.

6. Clarke LP, Velthuizen RP, et al. MRI stability of three supervised segmentation techniques. Magn Reson Imaging 1993; 11:95-106.

7. Cline HE, Dumoulin Cl, Hart Jr HR, et al. 3D reconstruction of the brain from MRI using a connectivity algorithm. Magn Reson Imaging 1987; 345-349.

8. Cline HE, Lorensen WE, Ludke S, Crawford CR, Teeter BC. Two algorithms for the three-dimensional reconstruction of tomograms. Med Phys 1988; 15:320-327.

9. Dawson-Saunders B, Trapp RG. Basic and Clinical Biostatistics. Norwalk:Appleton \& Langes, 1990; 79-99.

10. Rao SM. Neuropsychology of multiple sclerosis: a critical review. J Clin Exp Neuropsych 1986; 8:503-542.

11. Haughton VM, Yetkin FZ, Rao SM, et al. Quantitative MR in the diagnosis of multiple sclerosis. Magn Res Med 1992; 26:71-74.

12. Gonzalez CF, Mitchell DR, Sacchetti T, Seward JD, Knobler RL, Lublin FD. Correlation between structural brain lesions and emotional and cognitive function in patients with multiple sclerosis: an MRI study. Neuroradiology 1991 (suppl) 123124.

13. Mitchell DR, Swirsky-Sacchetti T, Knobler RL, Gonzalez CF, Seward J, Field HL, Santiago RS, Lublin FD. Analysis and correlation of mood state with cerebral MRI and severity of illness in patients with multiple sclerosis. Neurology 1991; 41 (suppl 1):145.

14. Sacchetti T, Mitchell DR, Seward JD, Gonzalez CF, Lublin FD, Fnobler RL, Field $H$. Neuropsychological and structural brain lesions in multiple sclerosis: a regional analysis. Neurology 1992; 42:1291-1295. 
15. Paty DW, Li DKB, The UBC MS/MRI Study Group, The IFNB MSII Study Group. MRI analysis results of a multicenter, randomized, double-blind, placebocontrolled trial. Neurology 1993; 43:655-661. 